UCRL-JC-127299

PREPRINT

\title{
3D Particle Simulations of Space-Charge-Dominated Beams in HIF Accelerator Experiments
}

\author{
D. P. Grote, A. Friedman, \\ S. M. Lund, I. Haber \\ This paper was prepared for submittal to the \\ 1997 Particle Accelerator Conference \\ Vancouver, B.C, Canada \\ May 12-16, 1997
}

May 9, 1997

This is a preprint of a paper intended for publication in a joumal or proceedings. Since changes may be made before publication, this preprint is made available with the underotanding that it will not be cited or reproduced without the permiesion of the anthor. 


\section{DISCLAIMER}

This document was prepared as an account of work sponsored by an agency of the United States Government. Neither the United States Government nor the University of California nor any of their employees, makes any warranty, express or implied, or assumes any legal liability or responsibility for the accuracy, completeness, or usefulness of any information, apparatus, product, or process disclosed, or represents that its use would not infringe privately owned rights. Reference herein to any specific commercial product, process, or service by trade name, trademark, manufacturer, or otherwise, does not necessarily constitute or imply its endorsement, recommendation, or favoring by the United States Government or the University of California. The views and opinions of authors expressed herein do not necessarily state or reflect those of the United States Government or the University of California, and shall not be used for advertising or product endorsement purposes. 


\title{
3D PARTICLE SIMULATIONS OF SPACE-CHARGE-DOMINATED BEAMS IN HIF ACCELERATOR EXPERIMENTS
}

\author{
D. P. Grote, A. Friedman, and S. M. Lund, LLNL, L-440 P. O. Box 808, Livermore, CA 94550 \\ I. Haber, NRL, Code 6790, Washington DC 20375
}

\section{Abstract}

The development of a high current, heavy-ion beam driver for inertial confinement fusion requires a detailed understanding of the behavior of the beam, including effects of the large self-fields. This necessity makes particle-in-cell (PIC) simulation the appropriate tool, and for this reason, the three-dimensional PIC/accelerator code WARP3d is being developed. WARP3d has been used extensively to study the creation and propagation of ion beams both to support experiments and for the understanding of basic beam physics. An overview of the structure of the code is presented along with a discussion of features that make the code an effective tool in the understanding of space-charge dominated beam behavior. A number of applications where WARP3d has played an important role is discussed, emphasizing the need of three-dimensional, first principles simulations. Results and comparisons with experiment are presented.

\section{INTRODUCTION}

The mainline approach of the U.S. for a driver for energy production by inertial confinement fusion is a heavy-ion induction accelerator. The beams used for heavy-ion fusion (HIF) have a large enough current that the space-charge effects dominate the behavior of the beam, in comparison to thermal effects. The necessity of including the significant space-charge makes theoretical analysis of the beam difficult, but makes the use of particle simulation techniques (used in plasma physics) ideal.

One- and two-dimensional particle-in-cell (PIC) codes have played a significant role in the understanding of space-charge dominated beam dynamics. Often though, full three-dimensional models are required to capture all of the relevant physics; there is much interaction of the longitudinal and transverse dynamics of the beams. Examples of cases where full-three dimensional simulation is necessary are the study of beam ends and longitudinal compression in a quadrupole lattice and the examination of various element misalignments and non-axisymmetric field errors. For these reasons, the three-dimensional PICl accelerator code WARP3d is being developed.

WARP3d[1] is a three-dimensional PIC code developed expressly for the purpose of simulating the behavior of high-current, space-charge dominated beams. The applications of interest are ongoing and future accelerator experiments in the HIF program and ultimately a full scale driver for inertial fusion energy power production. We are well on our way toward a source-to-target modeling capability needed for modeling a full scale driver.

\section{OVERVIEW OF WARP3d}

The WARP3d code combines the PIC technique with a description of the "lattice" of accelerator elements. In the PIC model, the calculation of the self-fields of the beam is done by solving Poisson's equation (i.e. electrostatic only) on a 3-D mesh which can move with the beam. Only electrostatic beam fields are important throughout most of an HIF accelerator since, with the large ion mass, the velocity is well below the speed of light, only reaching $\sim 0.3 c$ at the end. The particles are advanced using the full Lorentz equation, including the self electric field as well as the applied electric and magnetic fields. The beams can be created in their entirety at the start of the simulation or by injection. The injection can be off of a plane or a curved surface and can be at a set current or space-charge limited. Both steady-state and time-dependent simulations can be made. The steady-state algorithm is based on the algorithm used in EGUN[2].

The lattice is a general set of finite-length accelerator elements including quadrupoles for focusing, dipoles for bending, and induction gaps for acceleration, as well as more general elements with arbitrary fields. Another set of elements in the 3-D model specifies the locations and curvatures of bends. These are not physical elements but are the appropriate coordinate transformations needed to follow the beam around the bends. The calculation of the self-fields is also altered to include the curvature of this "warped" space.

The fields of the lattice elements can be specified at any of several levels of detail. At the lowest level, the lattice elements can be hard-edged with an axially uniform field over the extent of the element. Here, the "residence correction" method is used in the particle mover, scaling the applied field by the fraction of the velocity step spent inside the element, to ensure that the particles receive (to a good approximation) the correct impulse from each element independent of the timestep size and thus allowing larger timesteps to be used. The fields of elements may also be specified in more detail and more generally either via axially dependent multipolar field composition or via field description on a 3-D mesh. The electrostatic elements can alternately be implemented from "first principles" by self-consistently including the conductors in the field solution, using a subgrid-scale boundary resolution algorithm to afford a realistic description of the geometry.

WARP3d makes use of a number of modern programming practices such as use of an interpretive scripting language, coarse grained objects, and parallel processing. 


\section{BEND EXPERIMENT}

A recirculating induction accelerator has the potential of significantly reduced cost as a driver for heavy-ion fusion. There are a number of physics and engineering issues that still need to be examined. For that reason, the heavy-ion fusion group at LLNL has begun experiments leading up to a small scale recirculator which will have dimensionless parameters similar to those of a driver scale recirculator[3]. The bend experiment is the first step of the sequence and may be the first detailed examination of the behavior of a space-charge dominated beam in a bent lattice with quadrupole focusing.

In the current state of the experiment a matching section of seven electric quadrupoles is followed by eight permanent magnetic quadrupoles in a straight lattice, and then by five more magnetic quadrupoles which are in a bent lattice with electric dipoles between the quadrupoles to bend the beam. The total bend angle is $45^{\circ}$. The beam of singlycharged potassium ions is injected with an energy of 80 $\mathrm{keV}$, a current of $2 \mathrm{~mA}$, and a radius of $5.5 \mathrm{~mm}$. The space charge of the beam depresses the phase advance per lattice period from $70^{\circ}$ to $20^{\circ}$. The transport line is in total about five meters long.

The goals of the simulations are, in combination with experimental results, to understand the behavior of the beam in the bend and to fully characterize the beam to allow thorough understanding of what will be inserted into the completed ring.

Previous analytical work [4] and simulation[1] has shown that as a beam enters a bend, it experiences a growth in emittance which is related to the axial thermal spread of the beam. As a beam with a thermal spread enters a bend, the particles with different axial velocities will separate due to dispersion. This separation leads directly to an increase in RMS emittance, and as the nonlinear space-charge density due to the separation thermally mixes, the separation leads to an increase in the true emittance which is related to the beam temperature. In the length of the current $45^{\circ}$ bend experiment, only the increase in RMS emittance will be observed.

This growth in emittance can be used as an indirect measurement of the axial thermal spread in the experimental beam. The thermal spread is estimated from the temperature of the emitter to be $<0.1 \%$ of the axial velocity, too small to be measured directly. By comparing the emittance growth seen in the experiment with that of simulations, a better estimate can be obtained.

In most of the simulations, the beam is formed by injection from the aperture at the end of the diode. The field of the electric quadrupoles is obtained from a multipolar decomposition of the fields calculated from "first principles" in a separate WARP3d calculation including the geometry of the electric quadrupole conductors. The field from the magnetic quadrupoles is obtained analytically; knowing the arrangement of the permanent magnets which make up the quadrupoles, the field can be calculated and

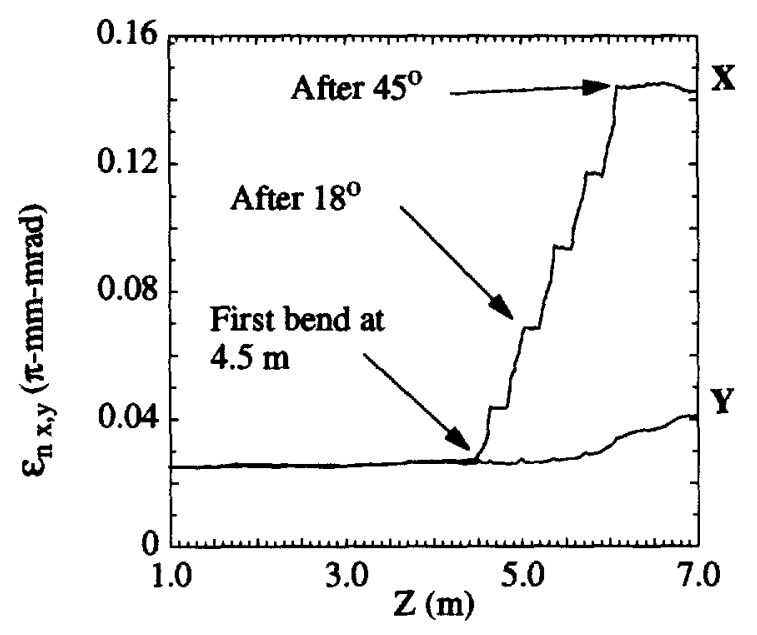

Figure 1: Normalized emittance at mid-pulse as the beam moves along the accelerator. The rise in the $x$ emittance is due to the axial thermal spread of the beam in the bend. The $y$ emittance begins to rise to match $x$. The thermal spread is $0.22 \%$ of the beam velocity.

then decomposed into multipolar components. The field from the dipoles is obtained by another first-principles solution of Laplace's equation including the carefullyshaped electric dipole plates. The field in a 3-D region around the plates is saved and applied directly to the particles.

Typically, 100,000 simulation particles and a $64 \times 32 \times 256$ field grid are used. About 800 timesteps are taken for the beam to travel completely though the lattice, taking 20 minutes on 32 processors of a Cray T3D.

With a thermal spread of $0.22 \%$ of the axial beam velocity, roughly the same as the transverse thermal spread, a significant emittance growth is seen as shown in figure 1. Figure 2 compares the transverse phase space in the plane of the bend before and after the bending. The spreading is very evident. In the plots, the particle shading is determined by the axial velocity, showing the separation of the different velocity classes. The particles with smaller $x$ ' are those with higher axial velocity; the beam is bending towards positive $x$ so the particles with higher axial velocity are bending less and thus have more negative $x$ and $x^{\prime}$.

A series of simulations was carried out with varying axial thermal spread. Figure 3 shows the final emittance after a $45^{\circ}$ bend versus the axial thermal spread. The increases in emittance for the velocity spreads shown are all well within acceptable limits for a beam to be injected into the ring. Note that for these runs, the fields of the matching electric quadrupoles were applied using the hard-edged approximation, to reduce the time of the simulation. Comparison with runs using the decomposed firstprinciples fields show little difference in the emittance growth.

Extensive simulations of the full small scale recirculator experiment have also been carried out. The simulation 

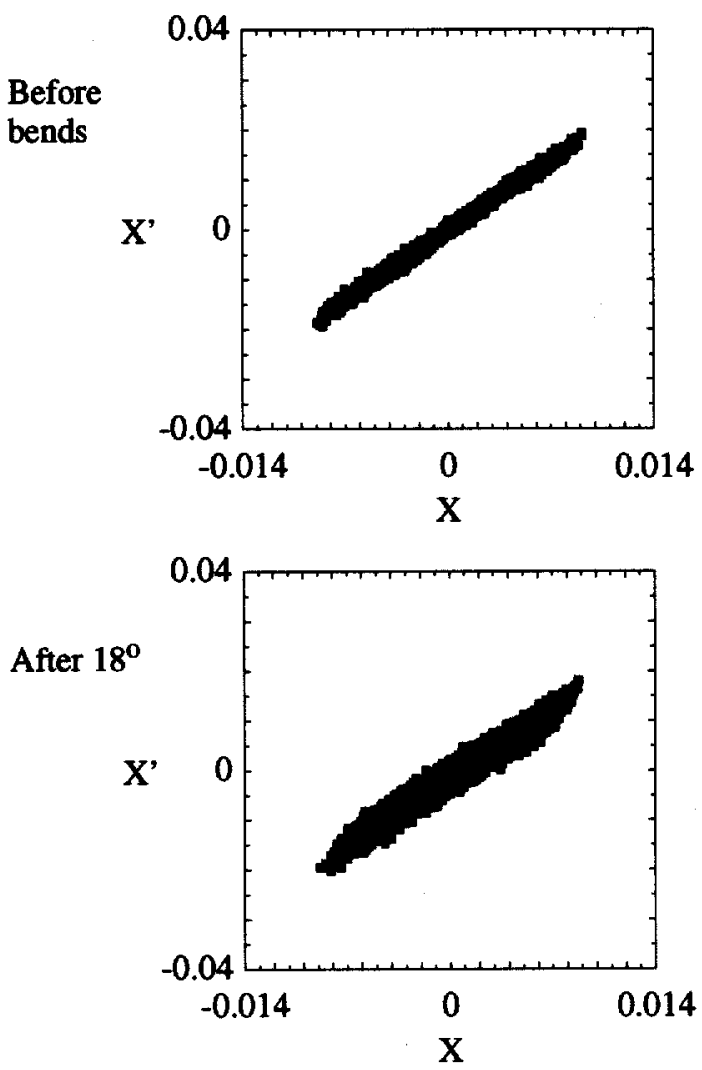

Figure 2: Phase space in the plane of the bend before and after an $18^{\circ}$ bend. The shading of the particles based on axial velocity shows the spreading from the bend. The particles with larger velocity have smaller $x^{\prime}$.

results show emittance growth from multi-lap operation that is well within acceptable limits. That work is discussed more fully in reference [1].

\section{MARYLAND BEND EXPERIMENT}

The University of Maryland has an experimental electron beam program that is examining the dynamics of high-current, low energy beams. Currently there exists a one meter long, $4 \mathrm{kV}, 20 \mathrm{~mA}$, injector test assembly[6]. In the planning stages is a ring[7]. The WARP3d code is beginning to be used in the examination of the experiments and in validation of the designs.

The focus to date has been on simulating the existing injector transport line. The focusing in the accelerator is obtained from printed circuit quadrupoles which were designed with a 3-D magnetics program. The fields from these quadrupoles are calculated in the 3-D magnetics program and are empirically fit to an analytic formula which is used in the simulation. Similarly, the fields from a solenoid, which is used to initially focus the beam as it comes off of the source, can be calculated and applied in the sim-

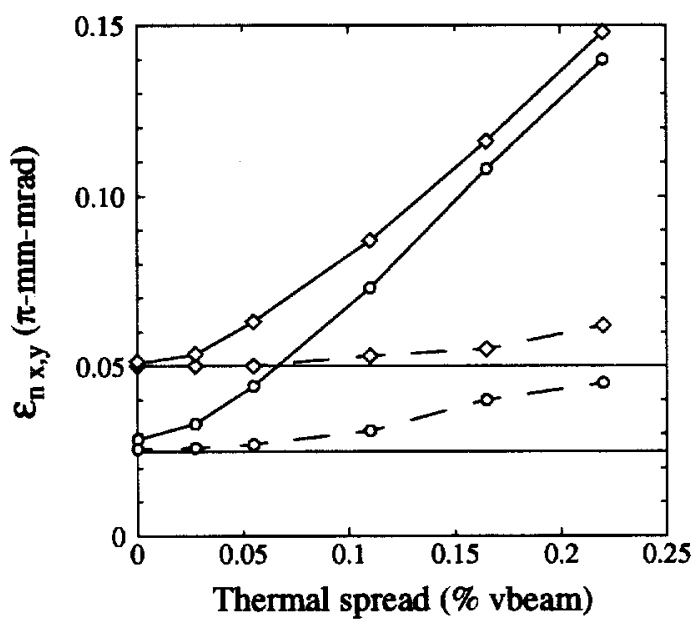

Figure 3: Final normalized emittance of the beam midpulse with changing axial thermal spread for two different initial emittances, 0.025 and $0.05 \pi$-mm-mrad. Solid line is in the plane of the bend, dashed is out of plane. The horizontal lines show the initial emittance. Comparison with the emittance from the experiment will give an indirect measurement of the axial thermal spread.

ulation. The calculated 3-D field structure can be applied directly in the simulation, but this has not been done yet. The simulated beam is created by continuous injection at a constant current at the end of the diode and is allowed to fill the system axially until a steady-state is reached.

As there is no experimental data yet, comparisons were made with another code, namely the envelope code SPOT[8]. Good agreement was found between the codes. There were minor differences, though, due in part to the fact that WARP3d allows a more complete description of the solenoid fields including the radially varying transverse fields. Use of the more complete description changes the effective focusing of the solenoid. The future plans are to use WARP3d to examine the design of the full electron ring. That work will rely heavily on the capability of WARP3d to model the transport of high-current beams around bends.

\section{ELECTROSTATIC QUADRUPOLE INJECTOR}

An injector for a driver-scale accelerator will be required to produce multiple beams with a high current. In order to examine the relevant physics and engineering issues, a driver scale injector experiment is being carried out at LBNL[5]. The injector design consists of a diode to extract a beam from a source, followed by a series of electric quadrupole focusing elements which have their voltages arranged to produce a net accelerating gradient down the axis.

The injector produces a $1 \mathrm{~A}$ beam of singly-charged potassium ions at $2 \mathrm{MeV}$. A major goal was production of a beam of low emittance. Since there are a number of issues in the design of the injector which can lead to emit- 
tance growth, an effort was started to study the injector with simulation. The major issue is the large size of the potentials of the electric focusing quadrupoles relative to the energy of the beam. That potential can cause a significant transverse variation of the axial velocity which distorts the beam, leading to emittance growth; this is referred to as the "energy effect".

The experiment has been simulated extensively using WARP3d. Initial simulations were of a smaller scale, proof of principle experiment and help demonstrate the feasibility of the injector design and improve upon it. The major improvement was knowledge of how much to increase the diode voltage. With that increase, the focusing potentials are a smaller fraction of the beam energy and the distortion due to the energy effect is less. Also, the beam is more easily focused and can be made smaller, further reducing the size of the distortion. The diode voltage is limited, though, on the upper end by breakdown considerations. Increasing the diode voltage from $500 \mathrm{kV}$ to 750 $\mathrm{kV}$ was shown by the simulations to significantly reduce the emittance growth while staying below the voltage breakdown threshold.

Self-consistent simulations of the full-scale experiment have been carried out[1]. The beam is created by spacecharge limited injection off the emitting surface. The full geometry of the diode region and of the electric quadrupole structure is included self-consistently, creating the applied fields and the image fields. Comparisons between simulation and experiment have shown excellent agreement in the beam size and divergence as well as in the shape of the beams transverse phase space.

The experimental results, though, have shown an unanticipated beam hollowing and non-linear transverse charge density distribution. Simulations of the experiment have eliminated such causes as the energy effect, and suggest that the hollowing could results from a nonuniform distribution generated in the diode. Studies of this phenomenon continue.

\section{BEAM COMBINER}

An optimal design of a driver may require the combining of multiple beams in order to take advantage of cost efficiencies of an architecture with many beams at low energy and few beams at high energy. The HIF group at LBNL is carrying out an experiment to examine beam combining[9]. The major issue is the increase in the emittance resulting from the filling of phase space between the beams when they are combined.

WARP3d is being used to simulate the beam transport. To date, the code has only been used for simulations of the matching section leading up to the combiner section. Fairly good agreement with the experimental results have been obtained. There is a small difference in the phase rotation which is likely due to uncertainties in the initial conditions which are used in the simulation. The simulations are shedding light on the cause of an unanticipated essing in phase space. These simulations are described in more detail in reference [10]. Our future plans include simulation of all of the experiment from the source through the combiner.

\section{AVLIS}

Though not within the HIF program, simulations for the atomic vapor laser isotope separation (AVLIS) program provide a means to further the use and utility of the WARP3d code. The AVLIS program is using an electron beam to melt and vaporize the material to be separated. The electron gun has shown anomalous asymmetric beam behavior that cannot be studied with axisymmetric codes. We have begun to use WARP3d to examine the gun with space-charge limited injection of the electrons off the emitter surface and use of magnetic fields calculated by TOSCA and specified on a 3-D grid. The full conductor geometry is simulated using subgrid-scale conductor resolution.

An additional focus of the simulations is placing tolerances on the construction of the gun diode. A series of simulations has been carried out offsetting various pieces of the diode to examine the degradation of beam quality. These simulations are made possible by the three-dimensional code.

\section{CONCLUSIONS}

The necessity of self-consistency in the self-field calculation and of full dimensionality in the study of the transport of high-current beams relevant for HIF has lead to the development of WARP3d, a three-dimensional particle-incell/accelerator code. Though this code is still under development, it has been successfully used in a wide range of applications, some of which have been described above. The major uses have been examination of beam dynamics in ongoing accelerator experiments being carried out by the U.S. HIF program. The code has been used to study, among other issues, injection, transport though lattices with non-linear fields, transport through bent lattices, and misalignments. WARP3d has played and continues to play an important role in the development of our understanding of high-current beam dynamics and in the analysis of high-current beam experiments.

\section{REFERENCES}

[1] D. P. Grote, et. al., "Three-Dimensional Simulations of High Current Beams in Induction Accelerators with WARP3d", Fus. Eng. \& Des., 32-33 (1996) 193-200.

[2] W. B. Herrmannsfeldt, "EGUN-An Electron Optics and Gun Design Program", technical report 331, SLAC, 1988.

[3] C. Sangster, "Status of the LLNL Experiments in Bending and Recirculating", this conference, 8V47.

[4] J. J. Bamard, H. D. Shay, S. S. Yu, A. Friedman, and D. P. Grote, "Emittance growth in Heavy Ion Recirculators", 1992 Linear Accelerator Conference Proceedings, Ottawa, Canada, vol. 1, p 229, AECL Research, (1992). 
[5] S. Yu, et. al., "Heavy Ion Fusion 2 MV Injector", in Proceedings of the 1995 Particle Accelerator Conference, Dallas, TX, pp 1178-82, IEEE, 1995.

[6] S. Bemal, et. al., "Transpont of Space-Charge Dominated Electron Beams in a Printed-Circuit Quadrupole Channel", this conference, 9V36.

[7] J. G. Wang, et. al., "Design and Development of an Electron Recirculator for Study of High Current Beam Dynamics", this conference, 6'C6.

[8] C. K. Allen, et. al., IEEE Cat. 95CH35843, 2324 (1996).

[9] P. A. Seidl, et. al., "A Scaled Beam-Combining Experiment for Heavy Ion Inertial Fusion", this conference, 8V46.

[10]W. M. Fawley, et. al., "Numerical Simulation Studies of the LBNL Heavy-Ion Beam Combiner Experiment", this conference, 8V45.

This work was performed under the auspices of the U.S. DOE by LLNL under contract no. H-7405-Eng-48. 


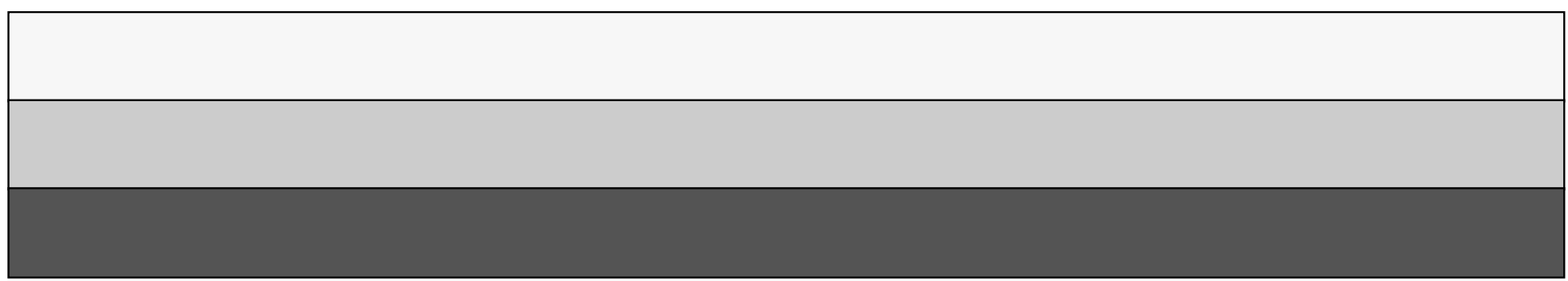

